\title{
EFECTO DE DIFERENTES MULCHINGS SOBRE EL CRECIMIENTO DE UNA PLANTACIÓN DE DURAZNEROS (Prunus persicae L.) VARIEDAD OPODEPE EN ZAVALLA, PROVINCIA DE SANTA FE
}

\author{
Flores, P.1; Ausilio, A. ${ }^{2}$; LeOne, A. ${ }^{1}$; Poggi, D. ${ }^{1 ;}$ Q Quadrelli, A. ${ }^{1}$; \\ Solini, I. ${ }^{2}$ LÓPeZ, M. ${ }^{1}$; Monti, F. ${ }^{2} \&$ Catraro, M. ${ }^{1}$
}

\begin{abstract}
RESUMEN
El objetivo de esta investigación fue determinar el efecto de mulchings orgánicos e inorgánicos sobre el crecimiento de durazneros var. Opodepe en el sur de la Prov. de Santa Fe. Las variables evaluadas fueron humedad de suelo, $\mathrm{pH}$, conductividad (CE) y crecimiento del cultivo. La humedad del suelo alcanzó los mayores valores en mulching orgánico, no existiendo diferencias significativas con respecto al inorgánico. No se registraron valores de $\mathrm{pH}$ que comprometieran el desarrollo de los durazneros. La CE aumentó en el mulching inorgánico, pero con valores no limitantes para el desarrollo del cultivo. Las variables de crecimiento mostraron los mejores resultados en mulching inorgánico. Este manejo asegura un eficiente establecimiento del cultivo porque permite un excelente control de las malezas sin necesidad de recurrir al control químico, ni mecánico, mientras que se recomienda utilizar mulching orgánico recién cuando el monte frutal está establecido.
\end{abstract}

Palabras clave: Prunus persicae, mulching orgánico, mulching inorgánico, control malezas.

\begin{abstract}
Effect of different mulchings in Prunus persicae var. Opodepe.

The objective was to determine the effect of mulchings on the growth of peach var. Opodepe in the south of Santa Fe. The variables evaluated were soil water content, $\mathrm{pH}$, electrical conductivity (EC) and crop growth. The soil water content reached the highest values in organic mulching, there being no significant differences with respect to inorganic. The registered $\mathrm{pH}$ values wasn't compro-
\end{abstract}

1.- Cátedra de Cultivos Intensivos. Facultad de Ciencias Agrarias, Universodad Nacional de Rosario. CC 14 (S2125ZAA) Zavalla, provincia de Santa Fe. E-mail: pflores@unr.edu.ar

2.- Cátedra de Edafología. FCA (UNR).

Manuscrito recibido el 28 de febrero de 2019 y aceptado para su publicación el 24 de junio de 2019.

Flores, P.; Ausilio, A.; Leone, A.; Poggi, D.; Quadrelli, A.; Solini, I.; López, M.; Monti, F. \& Catraro, M. Efecto de diferentes mulchings sobre el crecimiento de una plantación de durazneros (Prunus persicae L.) variedad Opodepe en Zavalla, Provincia de Santa Fe.

FAVE - Ciencias Agrarias 18 (1): 49-58. CC BY-NC-SA 4.0 (c) (7) (2) 


\section{P. Flores et al.}

mise the development of the peaches. EC increased in inorganic mulching, but with non-limiting values for crop development. The growth variables showed the best results in inorganic mulching. This management ensures an efficient establishment of the crop because it allows an excellent control of weeds without resorting to chemical or mechanical control, while is recommended to use organic mulching only when the fruit tree is established.

rs considered. So it is possible to conclude that TCQ inclusion in the conditions and dose used did not report any benefit in the parameters evaluated).

Key words: Prunus persicae, organic mulching, inorganic mulching, weed control.

\section{INTRODUCCIÓN}

El desarrollo de la industria química tuvo dos efectos, por un lado simplificó el trabajo del agricultor produciendo fertilizantes sintéticos de fácil aplicación y costo accesible (Sakovich, 1997), pero también provocó la contaminación de las aguas subterráneas por productos y residuos agroquímicos (FAO, 2002).

Según Ruiz y Febles (2004), el 52 \% de las tierras destinadas a la agricultura están moderada o severamente afectada por la degradación, perdiéndose entre 4 a 6 millones de hectáreas de suelo cultivado cada año. A esto se agrega el uso intensivo del suelo, con un excesivo laboreo para controlar las malezas, y la eliminación de la cubierta vegetal durante gran parte del año, desencadenándose el proceso erosivo (Goñi, 2007). Además, otras consecuencias negativas del mal uso del suelo son el aumento de la escorrentía, la disminución de la porosidad, la pérdida de la capacidad de infiltración del suelo y del almacenaje de agua, y la pérdida de materia orgánica, reduciéndose la fertilidad y la productividad de muchos cultivos (Allmaras et al., 2000; Bakker et al., 2004; Goñi, 2007; Wachs y Thibault, 2009; Brunel y Seguel, 2011).
En el presente, un cambio de actitud como consecuencia de la revalorización del medio ambiente, ha hecho que numerosos agricultores vuelvan a considerar los beneficios de agregar materia orgánica al suelo (Sakovich, 1997). La cobertura del suelo es el punto de partida para el desarrollo de estrategias de manejo sustentable, siendo las coberturas orgánicas e inorgánicas (mulchings) las prácticas recomendadas (Do Prado Wildner, 2000; Pinto de Oliveira y Marciano de Souza, 2003; Rienzi y Maggi, 2007; Goñi, 2007; Zribi et al., 2011). Se utilizan con mayor frecuencia materiales orgánicos como cortes de pasturas, rastrojos de cultivos, aserrín, cortezas y cascarillas de varios cereales, que se distribuyen en cualquier época del año, pero deben reponerse periódicamente porque se descomponen por acción de los microorganismos y del ambiente (Skroch et al., 1992; Anzalone Graci, 2008; Zribi et al., 2011; Valentini et al., 2012).

De acuerdo al concepto actual de la producción integrada, es recomendable utilizar materiales producidos localmente, por ejemplo en la región citrícola del río Uruguay emplean el chip de madera de eucalipto que es un material abundante porque son áreas forestadas con dicha especie (Gómez, 2015). 
Autores como Robinson (1988) y González y Amma (2012), recomiendan el uso de mulching orgánico porque favorece el crecimiento y vigor de las plantas por mayor conservación de la humedad del suelo, menor compactación, mejores temperaturas de suelo, y por el aporte de materia orgánica, manteniendo al suelo protegido evitando daños por erosión hídrica o eólica. También éste sistema de manejo brinda un buen comportamiento térmico en relación a las heladas, y se elimina la vegetación por ausencia de luz sin necesidad de aplicar herbicidas (Valentini et al., 2012). Sin embargo, es importante considerar que si los materiales utilizados tienen una elevada relación $\mathrm{C} / \mathrm{N}$, debería aplicarse alguna fuente de nitrógeno para evitar posible déficit de este nutriente en las plantas de duraznero. El mulching orgánico se recomienda utilizarlo como técnica mixta aplicada en la franja de plantación, y cuando el monte frutal lleva algunos años de implantado, manteniendo el resto de la superficie con cobertura vegetal, labores mecánicas o aplicación de herbicidas (González y Amma, 2012).

Los suelos con cubiertas orgánicas o inorgánicas, favorecen el desarrollo y la funcionalidad de las raíces, mejorando el intercambio gaseoso con la ventaja que además las raíces sobresalen hacia la superficie penetrando en el material de la cubierta si ésta es orgánica (Baldini, 1992).

Con respecto a los mulchings inorgánicos, la posibilidad de la reutilización de algunos materiales sintéticos, como el plástico del silo bolsa, constituye una buena alternativa en las plantaciones de frutales ya que aporta un doble beneficio, un efecto positivo en el cultivo y a su vez, contribuyendo a la disminución del impacto ambiental como consecuencia de su reutilización (Gómez, 2015).
Los materiales plásticos utilizados varían en cuanto a su espesor y color, destacándose el polietileno de baja densidad como el más usado para esta finalidad (Skroch et al., 1992; Tarara, 2000; Rodríguez Rodríguez, 2007; Verdú y Mas; 2007; Abouziena et al., 2008; Anzalone Graci, 2008; Zribi et al., 2011; Chen et al., 2012; Zhang et al., 2012; Goñi y Otero, 2012). Ciertamente es importante contribuir con el desarrollo económico de los productores frutícolas, atendiendo paralelamente a la importancia de las prácticas sustentables.

Por lo expuesto, es importante desarrollar experiencias locales, estudiando y adecuando éstas alternativas tecnológicas a diferentes cultivos intensivos de especies de interés socio-económico regional, como el duraznero.

El objetivo de este trabajo fue determinar el efecto de mulchings orgánicos e inorgánicos sobre el crecimiento de una plantación de duraznero (Prunus persicae L.) variedad Opodepe que se implantó en agosto de 2016 en la localidad de Zavalla, Provincia de Santa Fe.

\section{MATERIAL Y METODOS}

La investigación fue realizada durante los años 2016, 2017 y 2018 en una plantación de durazneros variedad Opodepe en el Campo Experimental Villarino de la Facultad de Ciencias Agrarias, (UNR) (latitud de $33^{\circ}$ $01^{\prime} \mathrm{S}$, longitud $60^{\circ} 53^{\prime} \mathrm{W}$ y $50 \mathrm{~m}$ de altura sobre el nivel del mar).

Previo al inicio del ensayo, en los meses de enero y abril, se aplicó glifosato LS $48 \%$ a una dosis de 1,04 kg i.a.ha-1 en todos los tratamientos para eliminar las malezas presentes. 
La plantación se realizó en el mes de junio del 2016, en un marco rectangular de plantación de $6 \mathrm{~m}$ entre filas y $4 \mathrm{~m}$ entre plantas, con riego suplementario y localizado por goteo. La frecuencia del riego se efectúo en función de lo que realizan normalmente los productores de la zona. En la estación de primavera la frecuencia fue de un riego semanal, y en verano dos riegos por semana, mientras que durante el otoño e invierno se regaba una vez al mes. La calidad del agua utilizada para el riego es de salinidad media, clasificada según el Laboratorio de Salinidad de los EEUU como C2 (Allison y Richards, 1962). El diseño experimental fue en bloques completamente aleatorizados con cuatro tratamientos y cuatro repeticiones. Los tratamientos fueron: T1) Mulching orgánico de 6 $\mathrm{cm}$ de espesor de chip de madera de frutales u otras especies; T2) Cobertura plástica (mulching inorgánico) de $250 \mu: 4.000 \mathrm{~m} 2 . \mathrm{ha}^{-1}$. Se utilizaron bolsas de silo de descarte. Se colocaron fajas de $1 \mathrm{~m}$ de ancho extendidas a ambos lados de la línea de troncos, unidas entre sí con grampas y sujetadas en los extremos con tierra. En los interfilares la maleza se controló con una motocultivadora; T3) Control químico en banda: aplicaciones en primavera, verano y otoño de herbicida glifosato (2 L.ha ${ }^{-1}$ ) y Diuron (4.00 L.ha-1) (manejo convencional de las quintas de la región; T4) Testigo: corte de la cobertura verde al ras con una motoguadaña.

El mulching orgánico utilizado fue chip de madera proveniente de la poda de árboles frutales y de algunos ornamentales del Parque Villarino (Localidad de Zavalla, Prov. de Santa Fe). El propósito fue utilizar materiales locales de acuerdo al concepto de producción integrada, con el beneficio del bajo costo que implica el triturado (chipeado) de las ramas, como se hace en otras regiones frutícolas de nuestro país donde utilizan chip de madera de eucalipto (Gómez, 2015).

Para el control de malezas en el interfilar se realizaron 2 pasadas de desmalezadora en primavera y otras dos en verano. El riego se realizó con una frecuencia semanal durante las estaciones de primavera y verano.

Las variables evaluadas fueron humedad de suelo, $\mathrm{pH}$, conductividad y crecimiento del cultivo. La humedad del suelo se determinó efectuando determinaciones gravimétricas. Habiendo apartando previamente la cobertura, se recolectaron 3 sub-muestras (una por árbol) a $10 \mathrm{~cm}$ de profundidad y a $30 \mathrm{~cm}$ de la línea del tronco. Cada muestra se colocó en una cápsula de aluminio con tapa identificada y se almacenó en cajas de tergopol para su transporte hasta el laboratorio. Se pesaron para determinar su peso fresco húmedo y se colocaron en estufa por 48 horas a $105{ }^{\circ} \mathrm{C}$ hasta peso constante para obtener su peso seco (ISO, 1993). Se midió el pH en agua $(1: 2,5)$ a través del método electrométrico (Vázquez, 2005). Para determinar la influencia de los diferentes tratamientos respecto a la variación del contenido salino, se utilizó el método de conductividad eléctrica por puente salino de Wheatstone (USDA, 1996), realizando un muestreo estacional.

El crecimiento del cultivo se evaluó cada seis meses midiendo la altura y diámetro de copa de la planta, diámetro del portainjerto e injerto, según la metodología propuesta por Pérez-Zamora et al. (2002).

Para la medición del diámetro del portainjerto e injerto (tronco) se utilizó un calibre, realizando las observaciones en sentido perpendicular a las líneas de plantación sobre una marca permanente ubicada a $10 \mathrm{~cm}$ del suelo para el portainjerto y a $15 \mathrm{~cm}$ por encima de la unión del injerto para este último. La altura de las plantas y el diámetro de las copas se registraron mediante cinta métrica. 
Los resultados obtenidos fueron analizados estadísticamente mediante el análisis de varianza y la comparación de medias mediante test de Tukey con nivel de significación de 0,05 , utilizando el software InfoStat (Di Rienzo et al., 2011).

\section{RESULTADOS Y DISCUSIÓN}

Para el tratamiento con mulching orgánico la humedad del suelo alcanzó los mayores valores, no existiendo diferencias significativas con relación al mulching inorgánico durante los meses de verano, pero sí respecto al testigo y al tratamiento con herbicida (Tabla 1).

Tal como afirman otros autores, éstos resultados se debieron a que en los tratamientos con coberturas disminuyó la evaporación de agua de las capas superficiales del suelo por una menor captación de radiación solar en relación al suelo desnudo (Robinson, 1988, Baldini, 1992; Zribi et al., 2011; González y Amma, 2012). Si bien la cubierta plástica obstaculiza la penetración del agua de lluvia por la impermeabilidad del material, es también eficiente para el control de la evaporación del suelo y el crecimiento de las malezas (Baldini, 1992).

Por otra parte, las elevadas precipitaciones registradas entre los meses de enero y julio de 2017 ocasionaron que en la primavera de ese año, por única vez, el tratamiento químico y el testigo acumularan mayores valores de humedad que el tratamiento con mulching inorgánico (Tabla 1). En cambio, en la campaña 2018 no se observó la misma situación para el mismo período, ya que no sólo se registraron menores precipitaciones, sino que éstas se concentraron entre los meses de abril y mayo, luego de una intensa sequía estival (Tabla 2).

Tabla 1: Humedad volumétrica del suelo a $10 \mathrm{~cm}$ de profundidad registrada en invierno y verano en los diferentes tratamientos de las campañas 2016, 2017 y 2018.

\begin{tabular}{|l|c|c|c|c|}
\hline & $\begin{array}{c}\text { Verano 2016 } \\
(\%)\end{array}$ & $\begin{array}{c}\text { Invierno 2017 } \\
(\%)\end{array}$ & $\begin{array}{c}\text { Verano 2017 } \\
(\%)\end{array}$ & $\begin{array}{c}\text { Invierno 2018 } \\
(\%)\end{array}$ \\
\hline M. Orgánico & $16,73 \mathrm{a}$ & $24,66 \mathrm{a}$ & $18,07 \mathrm{a}$ & $22,79 \mathrm{a}$ \\
\hline M. Inorgánico & $15,79 \mathrm{a}$ & $19,14 \mathrm{c}$ & $17,66 \mathrm{a}$ & $19,92 \mathrm{~b}$ \\
\hline T. químico & $10,58 \mathrm{~b}$ & $21,43 \mathrm{~b}$ & $15,1 \mathrm{~b}$ & $16,19 \mathrm{c}$ \\
\hline Testigo & $10,28 \mathrm{~b}$ & $21,7 \mathrm{~b}$ & $14,63 \mathrm{~b}$ & $15,57 \mathrm{c}$ \\
\hline F & 7,02 & 14,91 & 19,73 & 76,50 \\
\hline
\end{tabular}

Letras diferentes en las filas y columnas indican diferencias significativas dentro de cada estación para $p<0.05$.

Tabla 2: Registro de precipitaciones en milímetros para las campañas 2017 y 2018 en el periodo de enero a julio.

\begin{tabular}{|l|l|l|l|l|l|l|l|}
\hline Año & Enero & Febrero & Marzo & Abril & Mayo & Junio & Julio \\
\hline 2017 & 106 & 50 & 75 & 136 & 134 & 9 & 45 \\
\hline 2018 & 5 & & & 171 & 255 & 12 & 19 \\
\hline
\end{tabular}

Fuente: Agricultores federados argentinos AFA http://clima.afascl.coop/historico/?id=0004 
Con respecto al $\mathrm{pH}$, en ninguno de los tratamientos, y en ambas campañas, se registraron valores que comprometan el desarrollo adecuado del duraznero. Todos han sido inferiores a 7.5 , por lo que no se manifestaron síntomas de carencias nutricionales, especialmente la del hierro, que suele ser un problema frecuente en suelo con valores de $\mathrm{pH}$ superiores al mencionado (Baldini, 1992; Gariglio et al., 2007; Gariglio et al., 2014). Tampoco hubieron valores inferiores a 5.5 que comprometan la absorción de otros elementos como el nitrógeno, fósforo y potasio (Baldini, 1992). En general, los frutales crecen adecuadamente con valores de $\mathrm{pH}$ del suelo entre 5.5 y 8.5 , siendo para duraznero lo ideal un rango comprendido entre 6 a 7.5 (Gratacós, 2002; Gariglio et al., 2007).

En el tratamiento con mulching orgánico, debido a que la incorporación de materia orgánica libera ácidos, y actúa como buffer regulando los cambios de $\mathrm{pH}$, se registró estabilidad en los valores (Porta Casanellas et al., 2008). Mientras que en el tratamiento con mulching inorgánico se observó un leve incremento, pero por debajo del valor considerado perjudicial para la especie (Gratacós, 2002).
El $\mathrm{pH}$ puede verse aumentado por un incremento en la CE, dependiendo del tipo de sal que predomine en el agua de riego. Este efecto se manifiesta en mayor medida en el mulching inorgánico por no estar afectado al lavado de sales hacia profundidad por las precipitaciones. Si bien estadísticamente hubieron diferencias significativas entre tratamientos, éstas no tienen relevancia agronómica (Ausilio, comunicación personal) (Tabla 3).

Se observó un aumento sostenido de la CE en el tratamiento con mulching plástico, pasando de $139.67 \mu \mathrm{mho.cm}^{-1}$ en marzo de 2017 a $263.92 \mu$ mho.cm $^{-1}$ en marzo de 2018, lo que representa aproximadamente un $89 \%$ de aumento. Sin embargo, los valores obtenidos fueron inferiores a los considerados una limitante para el desarrollo del cultivo si se considera que la CE de un suelo normal es de 0 a $500 \mu$ mho (SAMLA, 2004). Los demás tratamientos también se mantuvieron dentro de los valores normales de $\mathrm{CE}$, pero presentaron fluctuaciones dependiendo del régimen de precipitaciones registrados en las diferentes épocas del año.

Tabla 3: Variación de los valores de pH de suelo registrados en los diferentes tratamientos evaluados.

\begin{tabular}{|l|c|c|c|c|}
\hline & Verano 2016 & Invierno 2017 & Verano 2017 & Invierno 2018 \\
\hline M. Orgánico & $6,62 \mathrm{a}$ & $6,66 \mathrm{a}$ & $6,66 \mathrm{a}$ & $6,93 \mathrm{a}$ \\
\hline M. Inorgánico & $7,18 \mathrm{c}$ & $7,34 \mathrm{~b}$ & $7,34 \mathrm{~b}$ & $7,16 \mathrm{a}$ \\
\hline T. químico & $6,8 \mathrm{ab}$ & $6,97 \mathrm{ab}$ & $6,97 \mathrm{ab}$ & $7,11 \mathrm{a}$ \\
\hline Testigo & $7,03 \mathrm{bc}$ & $6,84 \mathrm{a}$ & $6,84 \mathrm{a}$ & $6,85 \mathrm{a}$ \\
\hline F & 9,39 & 4,73 & 4,73 & 0,94 \\
\hline
\end{tabular}

Datos promedios de cuatro repeticiones. Letras diferentes significan diferencias significativas dentro de cada fecha. Según ANOVA y Comparación de medias según test de Tukey con un nivel de significación de 0,05. 
En todas las campañas evaluadas la cobertura plástica proporcionó los mejores resultados para las variables de crecimiento en estudio, existiendo diferencias significativas respecto a los demás tratamientos. La excepción fue la variable altura de planta que en la campaña 2018 no presentó diferencias significativas entre tratamientos, si bien en el tratamiento con plástico la altura media fue un $14 \%$ superior a la del mulching orgánico (Figura 1).

El mulching orgánico no presentó diferencias significativas respecto al tratamiento químico y al testigo (Figura 1). Tal como lo han señalado otros autores (González y Amma, 2012), esto es debido a que en las coberturas orgánicas como la utilizada en este ensayo, con elevada relación carbono: nitrógeno (C:N) (más de 100), la disponibilidad de nitrógeno del propio material es insuficiente para aumentar las poblaciones de microorganismos responsables de la descomposición de su materia orgánica. Es así que, éste nitrógeno demandado debe ser suministrado por el suelo, resultando en una inmovilización del elemento y una menor disponibilidad inmediata para el cultivo (Wolstenholme et al., 1996). Esto puede solucionarse aplicando alguna fuente de nitrógeno para evitar un posible déficit en las plantas de duraznero (Valentini et al., 2012). Esa fuente de nitrógeno no necesariamente
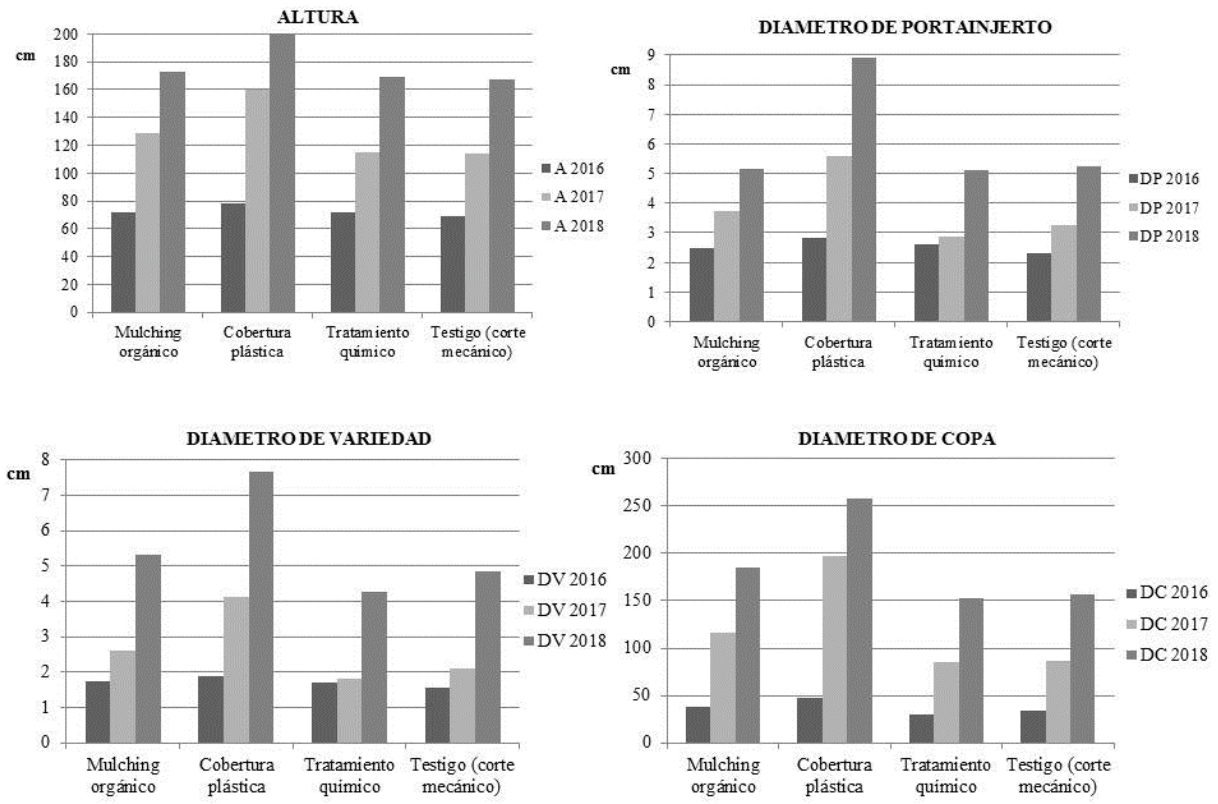

Figura 1: Efecto de cuatro tratamientos de manejo de suelo sobre la altura de la planta (A), diámetro de copa (DC), diámetro del portainjerto (DP) y diámetro del variedad (DV). Datos promedio de 4 repeticiones. 
debe ser un fertilizante químico, ya que puede utilizarse alguna otra fuente como estiércol de animales empleados en la producción orgánica (Zoppolo, 2014).

\section{CONCLUSIONES}

El mulching inorgánico asegura un eficiente establecimiento del cultivo del duraznero, ya que permite un excelente control de las malezas sin necesidad de recurrir al control químico, ni mecánico, contribuyendo a mantener un adecuado nivel de humedad del suelo.

Si bien el mulching orgánico favoreció la descomposición lenta de los residuos en contacto con la superficie del suelo aportando ácidos que mejoran sus condiciones fisicoquímicas, se recomienda comenzar a utilizarlo cuando el monte frutal está establecido.

\section{BIBLIOGRAFIA}

1.- ABOUZIENA, H.F; HAFEZ, O.M; EL-METWALLY, I.M; SHARMA, S.D. Y SINGH, M. 2008. Comparison of weed suppression and mandarin fruit yield and quality obtained with organic mulches, synthetic mulches, cultivation, and glyphosate. HortScience 43(3): 795-799.

2.- AGRICULTORES FEDERADOS ARGENTINOS (AFA). http://clima.afascl. coop/historico/?id=0004. Acceso: 18/06/19.

3.- ALLISON, L. Y RICHARDS, L. 1962. Diagnóstico y rehabilitación de suelos salinos y sódicos. Manual 60. Departamento de Agricultura de los EEUU. Limusa. Ciudad de México, México. 172p.
4.- ALLMARAS, R.R; SCHOMBERG H.H; DOUGLAS C.L. Y DAO, T.H. 2000. Soil organic carbon sequestration potencial of adopting conservation tillage in U.S. Coplands. J. Soil Water Conservation 55: 365-373.

5.- ANZALONE GRACI, A.L. 2008. Evaluación de alternativas al uso del polietileno como cubierta del suelo para el manejo de malas hierbas y otros aspectos agronómicos en el cultivo del tomate (Lycopersiconesculentum P. Mill.) en España y Venezuela. Tesis Doctoral. Universidad de Zaragoza. Zaragoza, España.

6.- BALDINI, E. 1992. Arboricultura Frutal. Ediciones Mundi Prensa. Madrid, España. 384pp.

7.- BAKKER, M.M; GOVERS, G. Y ROUNSEVELL, M.D.A. 2004. The crop productivity-erosion relationship: an analysis based on experimental work. Catena 57: 55-76.

8.- BRUNEL, N. Y SEGUEL, O. 2011. Efectos de la erosión en las propiedades del suelo. Revista Agro Sur 39(1): 1-12.

9.- CHEN, F; LIU, Y; TAO, Y; WAN, K.Y; ZHANG, G.S; LIU, D.B. Y XIONG, G.Y. 2012. Runoff and nutrient losses in citrus orchards on sloping land subjected to different surface mulching practices in the Danjiangkou Reservoir area of China. Agricultural Water Management. 110: 34-40.

10.- DO PRADO WILDNER, L. 2000. Cobertura del suelo. En: Manual de prácticas integradas de manejo y conservación de suelos. En: Boletín de tierras y aguas de la FAO No8. FAO. Roma, Italia. pp. 93-96.

11.- FAO. 2002. Agricultura mundial: hacia los años 2015/2030. Informe resumido. ISBN 92-5-304761-5. En: http://www.fao.org/3/ y3557s/y3557s00.htm. Acceso: 20/07/18.

12.- GARIGLIO, N.F.; PILATTI, R.A. Y AGUSTÍ FONFRÍA, M. 2007. Requerimientos ecofisiológicos de los árboles frutales. En: Sozzi, G. (Ed.) Árboles Frutales. Ecofisiología, Cultivo y Aprovechamiento. 
Editorial Facultad de Agronomía UBA. Buenos Aires, Argentina. p. 43-82.

13.- GARIGLIO, N. F.; C. A. BOUZO Y TRAVADELO, M.R. 2014. Cultivos frutales y ornamentales para zonas templado-cálidas. Experiencias en la zona central de Santa Fe. Ediciones UNL, colección Cátedra. Santa Fe, Argentina. 292 p. ISBN: 978-887-657-924-7.

14.- GRATACÓS, E. 2002. El Cultivo del Duraznero Prunus persica (L.) Batsch. Publicación de Universidad Católica de Valparaíso, Facultad de Agronomía, Cátedra de Fruticultura de Hoja Caduca, Valparaíso, Chile, 108p.

15.- GOMEZ, C. A. 2015. Efecto de distintos mulchings sobre la humedad y temperatura del suelo, estado hídrico, incidencia de malezas y crecimiento de un cultivo en implantación de mandarina Nova en Concordia, Entre Ríos. Argentina. Tesis Magister Scientiae en Cultivos Intensivos. Universidad Nacional del Litoral. Santa Fe, Argentina.

16.- GONZÁLEZ, J. Y AMMA, A.T. 2012. Manejo del suelo en monte de duraznero. En: Valentini, G.; González, J. yGordo, M. (eds.). Producción de duraznero en la región pampeana, Buenos Aires, Argentina. Ediciones INTA. 250 p. ISBN 978-987-679-124-3.

17.- GOÑI, M.C. 2007. Oportunidades para el manejo de suelos en cítricos, con una ópt ica de conservación y mejora. En: Avances de investigación en manejo y fisiología de cítricos. p. 26-33. Editores: GOÑI, M.C. \& OTERO, A. Programa Nacional Producción Citrícola. Actividades de difusión N$^{\circ}$ 516. INIA Salto Grande, República Oriental del Uruguay. pp. 34.

18.- GOÑI, M.C. Y OTERO, A. 2012. Citrus soil management con reduce the deterioration of soil conditions and improve sustainability production. Revista Agrociencia Uruguay 16(3): 89-97.ISO (International Organization for Standardization). 1993. Soil quality. Determination of dry matter and water content on a mass basis-Gravimetric method. ISO 11465:1993(E).
1: 3. En: https://www.iso.org/standard/20886. html. Acceso: 15/12/18.

19.- PÉREZ-ZAMORA, O; MEDINA-URRUTIA, V. Y BECERRA-RODRÍGUEZ, S. 2002. Crecimiento y rendimiento de naranja Valencia injertada en 16 portainjertos de cítricos establecidos en suelo calcimórfico, y calidad del jugo. Agrociencia 36: 137-148.

20.- PINTO DE OLIVEIRA, C.A. Y MARCIANO DE SOUZA, C. 2003. Influência da cobertura morta na umidade, incidência de plantas daninhas e de broca-do-rizoma (Cosmopolites sordidus) em um pomar de bananeiras (Musa spp.). Revista Brasileira de Fruticultura 25(2): 345-347.

21.- PORTA CASANELLAS, J.; LÓPEZ-ACEVEDO REGUERÍN, M. Y POCH CLARET, R.M. 2008. Introducción a la Edafología. Uso y Protección del Suelo. Ediciones Mundi Prensa. Madrid, España. ISBN: 9788484763420

22.- RIENZI, E.A. Y MAGGI, A.E. 2007. Manejo y conservación de suelos de sistemas frutícolas. En: Sozzi, G.O. (ed.) Árboles frutales: Ecofisiología, cultivo y aprovechamiento. Editorial Facultad de Agronomía - Universidad Nacional de Buenos Aires. Primera edición. Buenos Aires, Argentina. pp. 345-360.

23.- ROBINSON, D. 1988. Mulches and herbicides in ornamental plantinigs. Hortscience 23: 547-552.

24.- RODRÍGUEZ RODRÍGUEZ, G. 2007. Efecto de la cobertura del suelo con cascarilla de arroz en el crecimiento y rendimiento del tomate de ramillete. Revista Ciencia e Investigación Agraria 34(3): 225-230.

25.- RUIZ, T.E. Y FEBLES, G. 2004. La desertificación y la sequía en el mundo AIA Vol. 8-2 p. 3

26.- SAKOVICH, N. 1997. Soil organic matter. What it is and why you should use it. California Grower 21(3): 41-42. 
27.- SAMLA. 2004. Sistema de Apoyo Metodológico a Laboratorios de Análisis de suelos, aguas, vegetales y enmiendas orgánicas. SAMLA, primera edición. CD Rom. ISBN 987-918440-8.

28.- SKROCH, W.A; POWELL, M.A; BILDERBACK, T.E. Y HENRY, P.H. 1992. Mulches: durability, aesthetic value, weed control, and temperature. Journal of Environmental Horticulture 10(1): 43-45.

29.- TARARA, J.M. 2000. Microclimate modification with plastic mulch. HortScience 35(2): 169-180.

30.- USDA. 1996. United States Department Of Agriculture. 1996. Soil survey laboratory methods manual. Soil Survey Investigations Report $\mathrm{N}^{\circ}$ 42. Version 3.0. Washington DC, USA, 693p.

31.- VALENTINI, G.H; GONZÁLEZ, J. Y GORDO, M. 2012. Producción de duraznero en la región pampeana, Argentina. 1a ed. Buenos Aires, Argentina. Ediciones INTA. 250 p. ISBN 978-987-679-124-3.

32.- VAZQUEZ, M. 2005. Acidez del Suelo. En: Tecnologías en Análisis de Suelos. . En: Marban, L y Ratto, S. (eds.) Asociación Argentina de la Ciencia del Suelo, Buenos Aires, Argentina. pp. 69-88.

33.- VERDÚ, A.M. Y MAS, M.T. 2007. Mulching as an alternative technique for weed Management in mandarin orchard tree rows. Journal of Agronomy for Sustainable Development 27: 367-375.
34.- WACHS, T. Y THIBAULT, M. 2009. Beneficios de la gestión sostenible de la tierra. WOCAT (World Overview of Conservation Approaches and Technologies). Centre for Development and Environment, University of Berne. pp 16. [Online] http://www.unced. int/Lists/SiteDocumentLibrary/Publications/ CSD_Cover_Benefits_SLM\% 20spanisch. pdf. Acceso: 03/03/18.

35.- WOLSTENHOLME, B.N; MOORE-GORDON, C; ANSERMINO, S.D. 1996. Some pros and cons of mulching avocado orchards. South African Avocado Growers' Association Yearbook 19: 87-91.

36.- ZHANG, X; SUN, H; SHAO, L; LIU, X; MIAO, W; CHEN, S. 2012. Determination of water consumption and the water-saving potential of three mulching methods in a jujube orchard. European Journal of Agronomy 43: 87-95.

37.- ZOPPOLO, R. 2014. Programa Nacional de Investigación en Producción Frutícola. En: Manual Del Duraznero. La Planta Y La Cosecha. Boletín de divulgación 108. INIA. Las Brujas, Chile. pp. 255-279 [Online] http://www.ainfo.inia.uy/digital/bitstream/ item/8752/1/bd-108-p.255-279.pdf. Acceso: 18/06/19.

38.- ZRIBI, W; FACI, J.M; ARAGÜES, R. 2011. Efectos del acolchado sobre la humedad, temperatura y salinidad de suelos agrícolas. Información Técnica Económica Agraria 107(2): 148-162. 